Japan. J. Med. Sci. Biol., 30, 19-23, 1977

\title{
NOTES
}

\section{PREPARATION OF TOXOIDS FROM THE VENOMS OF PAKISTAN SPECIES OF SNAKES \\ (NAJA NAJA, VIPERA RUSSELLII AND ECHIS CARINATUS)}

The rural population of and the army in strategic movements in Pakistan are very much exposed to the hazards of snake bites. Snake-bite deaths occur 10,000 to 12,000 annually (Minton, 1968). They are mostly due to Cobra (Naja naja), Russell's viper (Vipera russellii), saw scaled viper (Echis carinatus) and kraits (Bungarus cerealis and B. candidus). There are no protective measures against venomous effects of the bites of these snakes except post-exposure therapy with the hyperimmune serum and other supportive therapy. The Biological Production Division of the National Health Laboratories, Islamabad, manufactures monovalent and polyvalent hyperimmune sera to the above-named snake venoms for post-exposure therapy. Serotherapy has its own limitations. It is effective only when applied soon after the snake bite before manifestation of tissue damage. In most of the cases it is too late when the antivenin is available to the victim of the snake bite. Moreover the serum reactions occurring in certain cases have their own hazards. Therefore, active immunization is required to protect people who are prone to the hazards of the snake bites. Toxoid(s) of the snake venom(s) could be used for active immunization.

Preparation of toxoid of snake venom involves complete detoxification of the venom and at the same time retention of immunogenicity of the product at its maximum. As pointed out by Kondo et al. (1971), attempts at toxoiding venoms by treatment with formalin or other chemicals or by irradiation with $\mathrm{X}$-ray and photo-oxidation made by a number of workers had only partial success, as detoxification was attained but the immunogenicity was impaired. Present attempts at preparation of highly immunogenic toxoids of three kinds of venoms by treatment with increasing concentrations of formalin following the methods of Sadahiro et al. (1970) and Kondo et al. (1971) are being reported.

Pools of lyophilized venoms of cobra (Naja naja), Russell's viper (Vipera russellii) and Echis carinatus were used. The lethal fraction of cobra venom (fraction pool III) was obtained by column chromatography on CM-cellulose as reported earlier (Khan et al., 1974).

The lethal toxicity of all the three venoms and the lethal fraction was determined by intravenous injection into mice of $16-18 \mathrm{~g}$ body weight and expressed in terms of $\mathrm{LD}_{50}$; the hemorrhagic activity was determined by intracutaneous injections into deplited skin of rabbits and expressed in terms of minimum hemorrhagic dose (MHD) following the methods of Kondo et al. 
(1960), Ohsaka (1960) and Khan et al. (1973, 1974).

Each of the crude venoms of cobra, Russell's viper and Echis carinatus was dissolved in $30 \mathrm{ml}$ of phosphate buffered saline $(\mathrm{pH} 7.0)$ to make a $1 \%$ solution. Detoxification of the venom solution was carried out following Sadahiro et al. (1970) and Kondo et al. (1971). Aliquots of venom solutions were treated with formalin at $0.2 \%, 0.4 \%, 0.6 \%$ and $0.8 \%$. The final formalin concentrations were attained by increasing concentration by $0.2 \%$ on every alternate day. Similarly the lethal fraction pool No. III of cobra venom was treated with formalin by increasing concentration by $0.2 \%$ finally reaching the maximum level of $0.8 \%$. The formalinized venom solution was incubated at $37 \mathrm{C}$ for a maximum period of 21 days with $\mathrm{pH}$ maintained at 7.0 with $\mathrm{NaOH}$. The extent of detoxification was determined on the $3 \mathrm{rd}, 5 \mathrm{th}, 7 \mathrm{th}, 10 \mathrm{th}, 14 \mathrm{th}$ and 21 st days after the 1st addition of formalin. For the purpose, a part of the formalinized solution (crude venom toxoid) and also that of cobra fraction III toxoid were dialyzed in cellophane tubing at 2-4 C against buffered saline ( $\mathrm{pH} 7.0$ ) to remove excess of formalin. The toxicities of both dialyzed and undialyzed solutions were determined by intravenous injections into mice of 16 to $18 \mathrm{~g}$ body weight.

Standard antivenins (monovalent) prepared by the Biological Production Division of the National Health Laboratories, Islamabad, against cobra, Russell's viper and Echis carinatus venom were used as the reference for the titration of circulating antitoxin of the animals immunized with the toxoids. Neutralization experiments were carried out with monovalent antisera of cobra, Russell's viper and Echis carinatus and the respective venom specimens, according to the instruction by Ipsen (1942). The unitage for each standard preparation was assigned arbitrarily 200 units per $\mathrm{ml}$.

The potency of a serum specimen to be tested was determined relatively to the corresponding standard preparation. Appropriate serum-venom mixtures were prepared with serial dilutions of both standard and test specimens and a constant amount of the corresponding venom. Groups of mice weighing 16-18 $\mathrm{g}$ were injected intravenously with $0.2-\mathrm{ml}$ doses each of the mixtures. The local standard for cobra antivenin was compared with the International Standard for Naja naja antivenin which was established in 1964. The potency was calculated as 200 units per $\mathrm{ml}$ in terms of the International Units by the neutralization method described above.

The time to death occurring within a period between $24 \mathrm{hr}$ up to 5 days was utilized for the calculation, since mice receiving injections with a mixture containing much free toxin (venom) die more rapidly than that containing a small amount of free toxin (venom).

Guinea pigs weighing $350-450 \mathrm{~g}$ and rabbits weighing $1.5-1.6 \mathrm{~kg}$ were immunized with each toxoid preparation according to the immunization schedules as mentioned in the legend to Table I. After the complete cycle of immunization, the animals were bled and circulating antitoxin titers were determined by the method stated above. The $\mathrm{ED}_{50}$ of each immune serum was determined by keeping the dose of respective venom constant and varying the dose of the serum 
and by calculating by the Reed and Muench method (1938). Each serum specimen from animals immunized with Russell's viper and Echis carinatus toxoids was titrated for anti-hemorrhagic activity (Kondo et al., 1965, 1971), while that from animals immunized with cobra venom was devoid of antihemorrhagic activity. The other groups of immunized animlas (guinea pigs and rabbits) were challenged 10 days after the last injection with 2-10 MLD of respective crude venom by intramuscular injections in the thigh muscles.

Gel diffusion tests on microscopic slides and precipitation tests in capillary tubes were also carried out for qualitative determination of corresponding antibodies in the sera of the immunized guinea pigs and rabbits with homologous and heterologous venom solutions. Positive controls were also maintained in all the tests using corresponding monovalent antivenin.

The snake venom solutions treated with formalin at concentrations ranging from 0.2 to $0.8 \%$ for a maximum period of 21 days resulted in detoxification at a concentration as low as $0.2 \%$. At concentrations of 0.6 and $0.8 \%$ detoxification was attained by 14 th and 10th days, respectively. The course of detoxification is depicted graphically (Fig. 1).

The circulating anti-toxin titers and $\mathrm{ED}_{50}$ of sera of immunized animals and the results of intramuscular challenging with venoms of cobra, Russell's viper and Echis carinatus are summarized in Table I. The gel diffusion and precipitation tests showed distinct precipitation bands for the toxoids of all the three venoms, whereas somewhat diffused precipitation bands were observed in

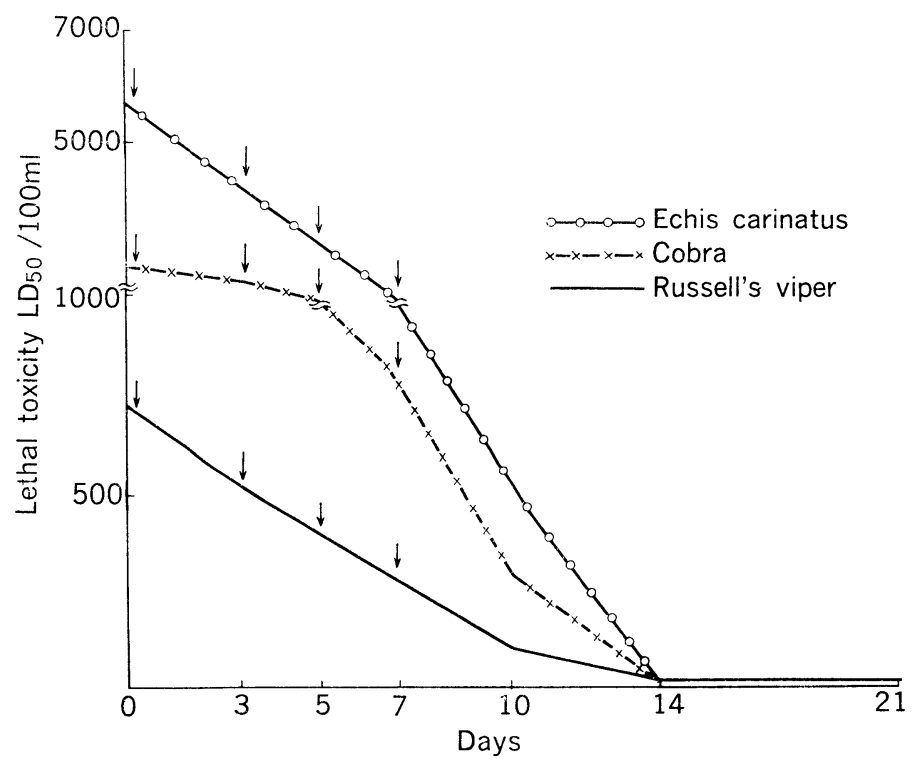

Fig. 1. * Detoxification schedule and time course of inactivation of lethal activity of Echis carinatus, cobra and Russell's viper venoms with formalin.

$\downarrow$ Addition of $0.20 \%$ formalin. 
TABLE I.

Circulating antitoxin titers and $\mathrm{ED}_{50}$ of sera of animals immunized with toxoids ${ }^{1)}$ and protection from intramuscular challenging with Cobra, Russell's viper and Echis carinatus venoms

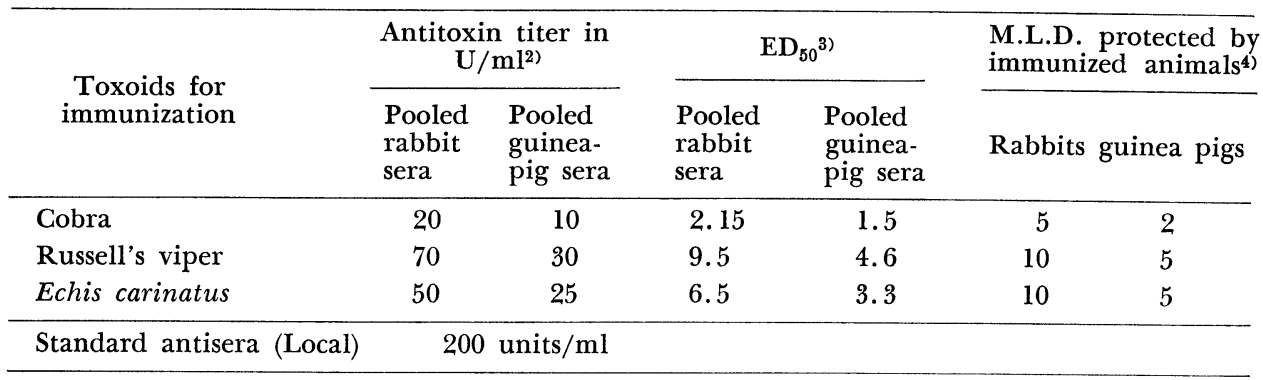

1) Schedule for immunization of rabbits and guinea pigs with toxoids. Subcutaneous injections on 1st, 3rd and 5th days in 1st, 3rd 5th weeks with alternate rest in 2nd and 4 th weeks. Immunizing doses were $0.2,0.2,0.3,0.3,0.5,0.5,1.0$ and $1.0 \mathrm{ml}$ for rabbits, and $0.1,0.1,0.2,0.2,0.3,0.3,0.3$ and 0.5 and $0.5 \mathrm{ml}$ for guinea pigs.

2) Separately pooled sera of four immunized rabbits and six immunized guinea pigs were titrated with crude venom.

3) The figures denote the number of $\mathrm{ED}_{50}$ of the Cobra, Russell's viper and Echis carinatus venoms neutralized with $1 \mathrm{ml}$ of the respective pooled sera of immunized rabbits and guinea pigs.

4) Protection from intramuscular challenging with 2.0 to $10 \mathrm{MLD}$ of the crude venoms to immunized rabbits and guinea pigs.

the case of serum from cobra-toxoid-immunized animals.

No side reactions were observed in the animals immunized with the toxoids. Immunized animals challenged with 2-10 MLD doses of corresponding venoms were protected as shown in Table I. It may be noted that Russell's viper antiserum has the capacity to neutralize $10 \mathrm{MHD}$ of the homologous venom, and Echis carinatus anti-serum neutralized $15 \mathrm{MHD}$ of its homologous venom.

The cobra venom fraction pool III was detoxified in the same way as was the crude cobra venom. In the gel diffusion test, clear precipitation bands could be observed at a dilution of $1 / 10$. The immunized rabbits could survive the challenge with up to only 5 MLD. The immunogenic response of guinea pigs was not exhibited at this challenge dose. The results obtained with toxoid to fraction pool III of cobra venom were nearly the same as those obtained with crude cobra-venom toxoid.

Detoxification of snake venoms irrespective of snake species were achieved on treatment with a low concentration of formalin and by incubation at $37 \mathrm{C}$ and $\mathrm{pH}$ 7.0. Formalin concentration and incubation period have inverse relationship as the lower the concentration, the longer the incubation period and with increasing the concentration the incubation time was shortened. This is in agreement with the finding of Sadahiro et al. (1970) and Kondo et al. (1971). The crude venoms detoxified with formalin at gradually increasing concentra- 
tions by $0.2 \%$ and finally reaching 0.6 and $0.8 \%$ have shown that the preparation retained the immunogenicity. The immunized animals survived the challenge with 5-10 MLD of respective venoms. The antisera against Russell's viper and Echis carinatus venoms neutralized 10-15 MHD of the respective venoms. The toxoid of the lethal fraction of cobra venom (Fraction pool III) was less immunogenic than crude venom toxoid.

\section{REFERENCES}

IPSEN, J. (1942): Systematisch und Zufallige Fehlerquellen bei Messung Kleiner Anti toxinmegen. Z. Immun.-Forscb., 102, 347.

Khan, Z. H., Jan, Z. A. ANd Ahmad, M. (1973): Fractionation of Russell's viper venom by chromatography on CM-cellulose with special reference to biological activities. A preliminary report. Japan. J. Med. Sci. Biol., 26, 39-43.

Khan, Z. H., Jan, Z. A., Zaidi, T. A. ANd FaruQui, A. Q. (1974): Fractionation of Cobra venom on CM-cellulose with special reference to biological activities. Japan. J. Med. Sci. Biol., $27,49-51$.

Kondo, H., Kondo, S., Ikezawa, H., Murata, R. and Ohsaka, A. (1960): Studies on the quantitative method for determination of hemorrhagic activity of Habu snake venom. Japan. J. Med. Sci. Biol., 13, 43-51.

Kondo, H., Kondo, S., Sadahiro, S., Yamauchi, K., Ohsaka, A. and Murata, R. (1965a): Standardization of antivenin. I. A method for determination of antilethal potency of $\mathrm{Habu}$ antivenine. Japan. J. Med. Sci. Biol., 18, 101-110.

Kondo, H., Kondo, S., Sadahiro, S., Yamauchi, K., Ohsaka, A. and Murata, R. (1965b): Standardization of antivenin. II. A method for determination of antihemorrhagic potency of Habu antivenine in the presence of two hemorrhagic principles and their antibodies. Japan. J. Med. Sci. Biol., 18, 127-141.

Kondo, S., Sadahiro, S., Yamauchi, K., Kondo, H. and Murata, R. (1971): Preparation and standardization of toxoid from the venom of Trimeresurus flavoviridis (Habu). Japan. J Med. Sci. Biol., 24, 281-294.

Minton, S. A., Jr. (1968): Snake Bite. p. 420-426. In Beeson, P. B. And McDermott [eds.], Cecil Loeb's Text Book of Medicine, 12th ed., W.B. Saunders Company, Philadelphia.

OHSAKA, A. (1960): Fractionation of Habu snake venom by chromatography on CM-cellulose with special reference to biological activities. Japan. J. Med. Sci. Biol., 13, 199-205.

ReEd, L. J. AND Muench, H. (1938): A simple method of estimating fifty percent end-points. Am. J. Hyg., 27, 493-497.

Sadahiro, S., Kondo, S., Yamauchi, K., Kondo, H. and Murata, R. (1970): Studies on immunogenicity of toxoid from Habu (Trimeresurus flavoviridis) venom. Japan. J. Med. Sci. Biol., $23,285-289$.

Biological Production Division, National Health Laboratories, Islamabad, Pakistan

(Received: April 3, 1976)
ZAHID HUSSAIN KHAN

Faiyaz Ahmed LARI

ZAFAR ALI 